\title{
Fuzzy Algorithm for Power Transformer Diagnostics
}

\author{
Nitin K. Dhote ${ }^{1}$ and Jagdish B. Helonde ${ }^{2}$ \\ ${ }^{1}$ St. Vincent Pallotti College of Engineering and Technology, Wardha Road, Nagpur 441108, India \\ ${ }^{2}$ Principal ITM College of Engineering, Nagpur, India
}

Correspondence should be addressed to Nitin K. Dhote; nitindhote@yahoo.com

Received 1 March 2013; Revised 13 May 2013; Accepted 28 May 2013

Academic Editor: M. Onder Efe

Copyright (c) 2013 N. K. Dhote and J. B. Helonde. This is an open access article distributed under the Creative Commons Attribution License, which permits unrestricted use, distribution, and reproduction in any medium, provided the original work is properly cited.

\begin{abstract}
Dissolved gas analysis (DGA) of transformer oil has been one of the most reliable techniques to detect the incipient faults. Many conventional DGA methods have been developed to interpret DGA results obtained from gas chromatography. Although these methods are widely used in the world, they sometimes fail to diagnose, especially when DGA results fall outside conventional methods codes or when more than one fault exist in the transformer. To overcome these limitations, the fuzzy inference system (FIS) is proposed. Two hundred different cases are used to test the accuracy of various DGA methods in interpreting the transformer condition.
\end{abstract}

\section{Introduction}

The power transformer is a vital equipment of the electrical power system. A transformer may function well externally with monitors, while some incipient deteriorations may occur internally to cause fatal problems in later development. Nearly $80 \%$ of faults result from incipient deteriorations. Therefore, faults should be identified and avoided at the earliest possible stage by some predictive maintenance technique. Dissolved gas analysis (DGA) is a reliable technique for detection of incipient faults in oil-filled power transformer. It is similar to a blood test or a scanner examination of the human body; it can warn about an impending problem, give an early diagnosis, and increase the chances of finding the appropriate cure. The working principle [1-4] is based on the dielectric breakdown of some of the oil molecules or cellulose molecules of the insulation due to incipient faults. When there is any kind of fault, such as overheating or discharge inside the transformer, it will produce corresponding characteristic amount of gases in the transformer oil. These gases are detected at the per part million (ppm) level by gas chromatography [5-10] It is a technique of separation, identification, and quantification of mixtures of gases. The commonly collected and analyzed gases are hydrogen $\left(\mathrm{H}_{2}\right)$, methane $\left(\mathrm{CH}_{4}\right)$, acetylene $\left(\mathrm{C}_{2} \mathrm{H}_{2}\right)$, ethylene
$\left(\mathrm{C}_{2} \mathrm{H}_{4}\right)$, ethane $\left(\mathrm{C}_{2} \mathrm{H}_{6}\right)$, carbon monoxide $(\mathrm{CO})$, and carbon dioxide $\left(\mathrm{CO}_{2}\right)$. Through the analysis of the concentrations of dissolved gases, their gassing rates, and the ratios of certain gases, the DGA methods can determine the fault type of the transformer. Even under normal transformer operational conditions, some of these gases may be formed inside.

Therefore, it is necessary to build concentration norms from a sufficiently large sampling to assess the statistics.

\section{DGA Interpretation}

If an incipient fault is present in the transformer, concentration of gases dissolved in the oil significantly increases. A given gas volume may be generated over a long time period by a relatively insignificant fault or in a very short time period by a more severe fault. Once a suspicious gas's presence is detected, it is important to be certain whether the fault that generated the gas is active by calculating the total dissolved combustible gases (TDCG) and rate of TDCG (10) which is given by

$$
R=\frac{\left(S_{T}-S_{0}\right) \cdot V \cdot 10^{-6}}{T}
$$


TABLE 1: Coding rule for IEC method [11].

\begin{tabular}{lccc}
\hline Codes & \multicolumn{3}{c}{ Range of gas ratios } \\
& $R 1$ & $R 2$ & $R 3$ \\
\hline 0 & $<0.1$ & $0.1-1$ & $<1$ \\
1 & $0.1-3$ & $<0.1$ & $1-3$ \\
2 & $>3$ & $>1$ & $>3$ \\
\hline
\end{tabular}

TABLE 2: Classification of faults by IEC method [11].

\begin{tabular}{llccc}
\hline $\begin{array}{l}\text { Fault } \\
\text { type }\end{array}$ & Characteristic fault & $R 1$ & $R 2$ & $R 3$ \\
\hline 1 & Normal ageing (N) & 0 & 0 & 0 \\
2 & Partial discharge (PD) of low energy density & 0 & 1 & 0 \\
3 & PD of high energy density & 1 & 1 & 0 \\
4 & Discharges of low energy (D1) & $1-2$ & 0 & $1-2$ \\
5 & Discharge of high energy (D2) & 1 & 0 & 2 \\
6 & Thermal fault of low temperature $(\mathrm{TL})<150^{\circ} \mathrm{C}$ & 0 & 0 & 1 \\
7 & TL between $150^{\circ} \mathrm{C}$ and $300^{\circ} \mathrm{C}$ & 0 & 2 & 0 \\
8 & TL between $300^{\circ} \mathrm{C}$ and $700^{\circ} \mathrm{C}$ & 0 & 2 & 1 \\
9 & Thermal fault of high temperature $(\mathrm{TH})>700^{\circ} \mathrm{C}$ & 0 & 2 & 2 \\
\hline
\end{tabular}

where $R$ is the rate (liters/day), So is the TDCG of first sample in ppm, $S_{T}$ is the TDCG of second sample in ppm, $V$ is tank oil volume in liters, and $T$ is the time (days).

The rate of generation of TDCG greater than 2.8 liters/day indicates that the transformer has an active internal fault and requires additional inspection by DGA methods.

Many interpretative methods employ an array of ratios of certain key combustible gases as the fault type indicators. These five ratios are

$$
\begin{aligned}
& R 1=\mathrm{C}_{2} \mathrm{H}_{2} / \mathrm{C}_{2} \mathrm{H}_{4}, \\
& R 2=\mathrm{CH}_{4} / \mathrm{H}_{2}, \\
& R 3=\mathrm{C}_{2} \mathrm{H}_{4} / \mathrm{C}_{2} \mathrm{H}_{6}, \\
& R 4=\mathrm{C}_{2} \mathrm{H}_{6} / \mathrm{C}_{2} \mathrm{H}_{2}, \\
& R 5=\mathrm{C}_{2} \mathrm{H}_{2} / \mathrm{CH}_{4} .
\end{aligned}
$$

Rogers' method [13-15] utilizes three ratios $R 1, R 2$, and $R 3$. The method gives fault for the specific combination of these gas ratios. Dornenburg [13-15] utilizes four ratios $R 1, R 2, R 4$, and $R 5$. This procedure requires significant levels of gases to be present for the diagnosis to be valid. The method gives fault after comparing these ratios to the limiting values.

Amongst ratio methods, IEC Standard 60599 [11] is most widely used. It also utilizes three ratios $R 1, R 2$, and $R 3$. The coding rule and classification of faults by the IEC method are given in Tables 1 and 2.

Incipient faults can be reliably identified by visual inspection [16] of the equipment after the fault has occurred in service as follows:

(a) PD-possible $\mathrm{X}$ wax formation and sparking inducing small carbonized punctures in the paper.

(b) D1-larger punctures in the paper, tracking, or carbon particles in oil. (c) D2-extensive carbonization, metal fusion, and possible tripping of the equipment.

(d) $\mathrm{TL}$-for $\mathrm{TL}<300^{\circ} \mathrm{C}$, the paper turns brown, for $\mathrm{TL}>$ $300^{\circ} \mathrm{C}$, the paper carbonizes.

(e) TH-oil carbonization, metal coloration, or fusion.

The Duval Triangle [17-19] method utilizes three \% ratios of certain gases for DGA interpretation of transformers filled with mineral oil. The triangular coordinates corresponding to DGA results in ppm can be calculated by (2) as follows:

$$
\begin{aligned}
{ }_{0} \mathrm{C}_{2} \mathrm{H}_{2} & =\frac{100 \cdot x}{(x+y+z)}, \\
\% \mathrm{C}_{2} \mathrm{H}_{4} & =\frac{100 \cdot y}{(x+y+z)}, \\
\% \mathrm{CH}_{4} & =\frac{100 \cdot z}{(x+y+z)},
\end{aligned}
$$

where $x, y$, and $z$ are concentrations of $\mathrm{C}_{2} \mathrm{H}_{2}, \mathrm{C}_{2} \mathrm{H}_{4}$, and $\mathrm{CH}_{4}$ in ppm, respectively. Along with three \% ratios given by Duval Triangle method, fourth \% ratio [12] is also used for fault diagnosis which is given by

$$
\% \mathrm{H}_{2}=\frac{100 \cdot \mathrm{H}_{2}}{\left(\mathrm{H}_{2}+\mathrm{C}_{2} \mathrm{H}_{6}+\mathrm{CO}+\mathrm{CO}_{2}\right)} .
$$

All these techniques are computationally straightforward. However, these methods in some cases provide erroneous diagnoses as well as no conclusion for the fault type.

To overcome these limitations, FIS is proposed.

\section{Diagnostic Procedure}

Flow chart of proposed system diagnosis is shown in Figure 1. The input data include concentration of dissolved gases $\mathrm{C}_{2} \mathrm{H}_{2}, \mathrm{C}_{2} \mathrm{H}_{4}, \mathrm{C}_{2} \mathrm{H}_{6}, \mathrm{CH}_{4}, \mathrm{H}_{2}, \mathrm{CO}$, and $\mathrm{CO}_{2}$ of the sample. Information such as tank oil volume, date of sampling, and date of installation of transformer is asked for further inference.

In the first step, the system calculates TDCG and compares with the standard permissible limits (IEEE standard, 2008). For normal level of TDCG $(<720 \mathrm{ppm})$, permissible limits for individual gases are checked. The normal level of TDCG and individual gases indicates the satisfactory operation of a transformer. Once an abnormal level of TDCG or individual gas has been detected, the next step is to determine the rate of generation of TDCG (1) by analysis of the successive sample. For the normal rate of TDCG (less than 2.8 liters/day), further diagnosis is bypassed. For an abnormal rate of TDCG, the proposed FIS is adopted to diagnose the probable faults. In the last step, severity degrees are assigned to the diagnosed faults. On the basis of severity of faults, appropriate maintenance actions are suggested.

\section{Fuzzy Inference System (FIS)}

Intelligent algorithms, for example, expert system [20], FIS [21, 22], artificial neural networks [23-25], probabilistic 


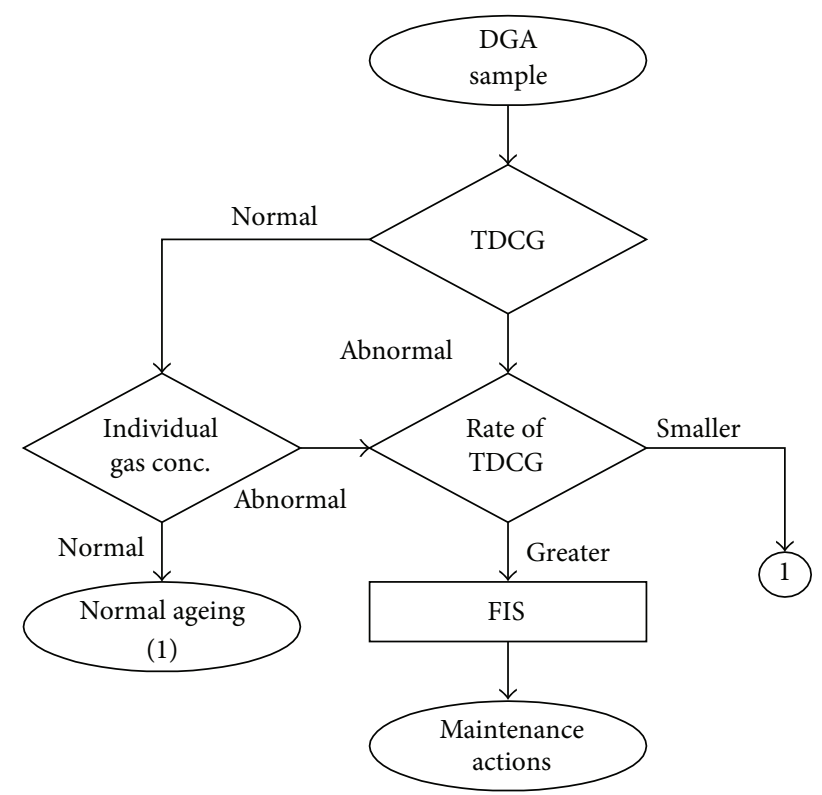

FIGURE 1: Flow chart of proposed system diagnosis.

neural networks [26], evolving neural networks [27], artificial neural FIS [28-31], wavelet networks [32], and combined neural networks and expert system [33] have been used to interpret DGA results. These algorithms are not entirely satisfactory. These methods are mostly suitable for transformers with single fault. In case of multiple faults, only dominant fault is indicated by these methods. These methods are based on specific set of codes defined for certain gas ratios. Further, no quantitative indication for severity of fault and maintenance suggestions is given by these methods.

The proposed fuzzy diagnostic method is prepared using the MATLAB Fuzzy Logic Toolbox [34]. Sugeno type FIS [3537] is used as a fuzzy inference method.

The rule for the zero-order Sugeno model is given below; If input $1=x$ and input $2=y$, then output $z=$ constant.

The output level $z$ of each rule is weighted by firing strength $w_{i}$ of the rule. For an AND rule, firing strength is given as

$$
w_{i}=\text { AND method }[F 1(x), F 2(y)],
$$

where $F 1(x)$ and $F 2(y)$ are the membership functions for input 1 and input 2 . The final output of the system is weighted average of all the rules output which is given by

$$
Y=\frac{\sum_{i=1}^{N} w_{i} z_{i}}{\sum_{i=1}^{N} w_{i}},
$$

where $Y$ is final output and $N$ is the number of rules.

The FIS consists of 3 ratios $R 1, R 2$, and $R 3$ as inputs. The coding rule for ratios is kept the same as IEC Method (Table 1). One of the major drawbacks of IEC method is that when gas ratio changes across coding boundary, the code changes sharply between 0,1 , and 2 . In fact the gas ratio boundary should be fuzzy. Depending on the relative values of ratios, IEC codes 0,1 , and 2 are replaced by fuzzy

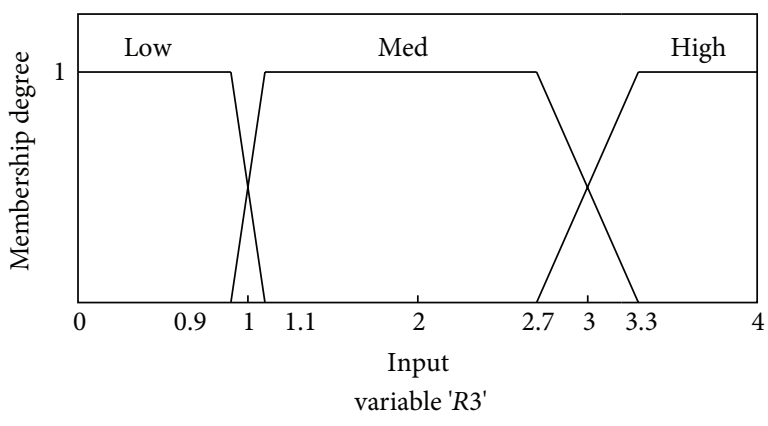

Figure 2: Membership function for ratio $R 3$.

codes Low, Medium (Med), and High. Due to uncertainty in measurements of gas concentrations by gas analyzers, the gas ratios would have a relative uncertainty of plus or minus $10 \%$ [38]. Membership function for code 0 of ratio $R 1$ is given by the linear declining function:

$$
\mu_{\text {Low }}(R 1)= \begin{cases}1 & \longrightarrow R 1 \leq 0.09 \\ \frac{(0.11-R 1)}{(0.11-0.09)} & 0.09 \leq R 1 \leq 0.11 \\ 0 & R 1 \geq 0.11\end{cases}
$$

Membership function for code 1 of ratio $R 1$ is given by trapezoidal function

$$
\mu_{\mathrm{Med}}(R 1)= \begin{cases}0 & \longrightarrow R 1 \leq 0.09 \\ \frac{(R 1-0.09)}{(0.11-0.09)} & \longrightarrow 0.09 \leq R 1 \leq 0.11 \\ 1 & 0.11 \leq R 1 \leq 2.7 \\ \frac{(3.3-R 1)}{(3.3-2.7)} & 2.7 \leq R 1 \leq 3.3 \\ 0 & R 1 \geq 3.3 .\end{cases}
$$

Membership function for code 2 of ratio $R 1$ is given by linear increasing function:

$$
\mu_{\text {High }}(R 1)= \begin{cases}0 & \longrightarrow R 1 \leq 2.7 \\ \frac{(R 1-2.7)}{(3.3-2.7)} & \longrightarrow 2.7 \leq R 1 \leq 3.3 \\ 1 & R 1 \geq 3.3\end{cases}
$$

The codes of the ratios $R 2$ and $R 3$ are also fuzzified as Low, Med, and High variable depending on the range of ratios for these codes. Membership function for ratio R3 is shown in Figure 2.

The FIS comprises of single output which has 5 fault types as membership functions. Weight in the range of 0 to 1 is assigned to each fault type on the basis of severity of the fault. The five types of faults used in FIS are TL $\langle 0.2\rangle, \mathrm{PD}\langle 0.4\rangle, \mathrm{D} 1$ $\langle 0.6\rangle, \mathrm{TH}\langle 0.8\rangle$, and D2 $\langle 1.0\rangle$.

The major drawback of the IEC method is that 16 IEC code combinations out the possible 27 do not indicate any fault. Only 11 inference rules are suggested by the IEC (Table 2$)$ out of the $27(3 \times 3 \times 3)$ possible rules. To overcome this limitation, existing 11 rules are modified in terms of fuzzy variables and additional 16 new rules are obtained as a 
TABLE 3: Comparison of accuracy of different methods.

\begin{tabular}{lcccc}
\hline & $T_{R}$ & $T_{P}$ & $A_{P}$ & $A_{C}$ \\
\hline Doernenburg & 77 & 111 & 69.37 & 38.50 \\
Roger & 89 & 145 & 61.38 & 44.50 \\
IEC & 142 & 170 & 83.53 & 71.00 \\
Duval Triangle & 172 & 200 & 86.00 & 86.00 \\
Li et al. [12] & 181 & 200 & 90.50 & 90.50 \\
Proposed method & 187 & 200 & 93.50 & 93.50 \\
\hline
\end{tabular}

result of extensive consultations with utility experts, existing literature, and approximately 1500 DGA case histories. Each rule consists of two components which are the antecedent (IF part) and the consequent (THEN part). The rules having a similar output are clubbed together and kept in order of increasing value of the severity of fault. The fuzzy rules are given below.

Rule 1. if $R 1=$ Low, $R 2=$ Low, $R 3=$ Med, then fault $=$ TL.

Rule 2. if $R 1=$ Low, $R 2=$ Med, $R 3=$ Low, then fault $=$ TL.

Rule 3. if $R 1=$ Low, $R 2=$ Med, $R 3=$ Med, then fault $=$ TL.

FIS derives output from judging all the fuzzy rules by finding the weighted average of all 27 fuzzy rules output.

\section{Case Studies, Results and Discussions}

FIS is developed based on the proposed interpretative rules and diagnostic procedure of an overall system. To demonstrate the feasibility of the system in diagnostic, 200 DGA gas records supplied by the major power companies in India have been tested.

Accuracy is calculated in two different ways as follows.

(a) When considering only the number of predictions, percentage accuracy is given by

$$
A_{P}=\frac{100 \cdot T_{R}}{T_{P}},
$$

where $T_{R}$ is the number of correct predictions and $T_{P}$ is the total number of the predictions.

(b) When considering the total number of cases, percentage accuracy is given in by

$$
A_{C}=\frac{100 \cdot T_{R}}{T_{C}},
$$

where $T_{C}$ is the total number of cases.

Accuracy values of different methods for total 200 cases are compared and summarized in Table 3. Results from three case studies are presented here.

5.1. Case Study-I. A $10 \mathrm{MVA}, 132 \mathrm{KV} / 11 \mathrm{KV}$ transformer is in service for 11 years. Tank oil volume is 12000 liters. On load tap changer inside main tank had intermittent sparking on some of the contacts. One nail was found on the shield of bottom tank, and few burns were observed on the nail and the bolt. DGA data obtained in ppm after the fault on 13/02/2009 is as follows: $\mathrm{C}_{2} \mathrm{H}_{2}-4 ; \mathrm{C}_{2} \mathrm{H}_{4}-54 ; \mathrm{CH}_{4}-09 ; \mathrm{H}_{2}-78 ; \mathrm{C}_{2} \mathrm{H}_{6}-67$; $\mathrm{CO}-670 ; \mathrm{CO}_{2}-1243$.

Step 1. TDCG in $\mathrm{ppm}=882$. TDCG is above normal (>720 ppm).

Step 2. The transformer is sampled again on $20 / 02 / 2009$ to determine rate of TDCG. Concentrations of dissolved gases in ppm are as follows: $\mathrm{C}_{2} \mathrm{H}_{2}-6 ; \mathrm{C}_{2} \mathrm{H}_{4}-73 ; \mathrm{CH}_{4}-14 ; \mathrm{H}_{2}-158$; $\mathrm{C}_{2} \mathrm{H}_{6}-75$; CO-831; $\mathrm{CO}_{2}-1430$.

TDCG in ppm = 1157; rate of TDCG $=4.71 \mathrm{lit} / \mathrm{day}$, which is greater than the normal level (2.8 lit/day).

Step 3. FIS is applied for fault diagnosis. The output of FIS is given by rule viewer which is shown in Figure 3. Rule viewer shows $R 1=0.082$ (Low), $R 2=0.088$ (Low), and $R 3=0.973$ which lies on the boundary of the fuzzy ratios Low and Med. Dark dots in the first and eighth rows of the fault column show that rules 1 and 8 are satisfied which indicates possible faults TL and PD, respectively. This result matches the actual fault of the transformer. Weighted average of both rules is given as 0.327 . Weight of both faults can be calculated as follows:

$$
\begin{gathered}
\text { weight of } \mathrm{TL}=\frac{(0.4-0.327)}{(0.4-0.2)}=0.365, \\
\text { weight of } \mathrm{PD}=1-0.365=0.635 .
\end{gathered}
$$

The weights point towards the strong possibility of fault PD and the relatively less possibility of fault TL. The key feature of the proposed method is that it can diagnose multiple faults unlike conventional DGA methods.

Step 4. Severity of faults is Medium. Maintenance actions suggested are as follows.

(1) Observe caution,

(2) Retest oil monthly,

(3) Determine load dependence.

5.2. Case Study-II. A $40 \mathrm{MVA}, 220 \mathrm{KV} / 11 \mathrm{KV}$ transformer is in service for 23 years. Tank oil volume is 28000 liters. This transformer had overheated off circuit tapping switching contacts. DGA data obtained in ppm after the fault on 11/06/2010 is as follows: $\mathrm{C}_{2} \mathrm{H}_{2}-31 ; \mathrm{C}_{2} \mathrm{H}_{4}-53 ; \mathrm{CH}_{4}-304 ; \mathrm{H}_{2}-163$; $\mathrm{C}_{2} \mathrm{H}_{6}-15$; CO-524; $\mathrm{CO}_{2}-786$.

Step 1. TDCG in ppm $=1090$. TDCG is above normal (>720 ppm).

Step 2. The transformer is sampled again on $14 / 06 / 2010$ to determine rate of TDCG. Concentrations of dissolved gases in ppm are as follows: $\mathrm{C}_{2} \mathrm{H}_{2}-34 ; \mathrm{C}_{2} \mathrm{H}_{4}-69 ; \mathrm{CH}_{4}-353 ; \mathrm{H}_{2}-197$; $\mathrm{C}_{2} \mathrm{H}_{6}-22$; CO-618; $\mathrm{CO}_{2}-931$.

TDCG in $\mathrm{ppm}=1292$; rate of TDCG $=18.85 \mathrm{lit} / \mathrm{day}$, which is greater than the normal level (2.8 lit/day). 


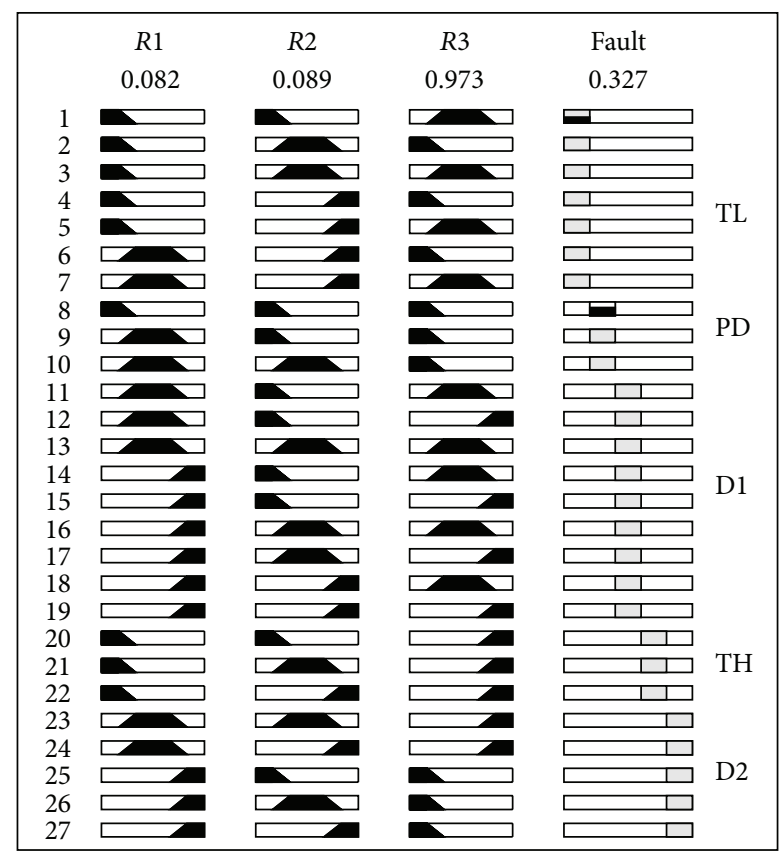

FIGURE 3: Rule viewer for case-I.

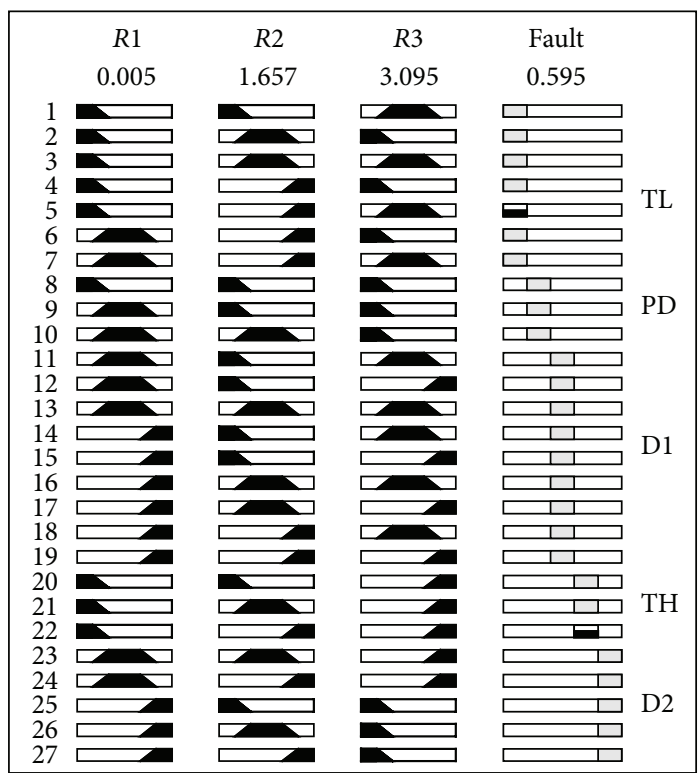

FIgURE 4: Rule viewer for case-II.

Step 3. FIS is applied for fault diagnosis. The output of FIS is given by rule viewer which is shown in Figure 4. Rule viewer shows $R 1=0.493$ (Med), $R 2=1.79$ (High), and $R 3$ $=3.14$ which lies on the boundary of the fuzzy ratios Med and High. Dark dots in the fault column show that rules 7 and 22 are satisfied which indicates possible faults TL and $\mathrm{TH}$, respectively. This result matches the actual fault of the transformer. Weighted average of both rules is given as 0.636. Weight of both faults can be calculated as follows:

$$
\begin{gathered}
\text { weight of } \mathrm{TL}=\frac{(0.8-0.636)}{(0.8-0.2)}=0.273 \\
\text { weight of } \mathrm{PD}=1-0.273=0.727
\end{gathered}
$$

The weights point towards the strong possibility of fault $\mathrm{TH}$ and the relatively less possibility of fault TL.

Step 4. Severity of faults is High. Maintenance actions suggested are as follows.
(1) Observe extreme caution.
(2) Retest oil weekly.
(3) Plan outage.

5.3. Case Study-III. A $25 \mathrm{MVA}, 220 \mathrm{KV} / 132 \mathrm{KV}$ transformer is in service for 15 years. Tank oil volume is 20000 liters. This transformer had an X - wax deposition. Traces of discharges were found on paper of high voltage cable. DGA data obtained in ppm after the fault on 18/03/2009 is as follows: $\mathrm{C}_{2} \mathrm{H}_{2}-15 ; \mathrm{C}_{2} \mathrm{H}_{4}-19 ; \mathrm{CH}_{4}-172 ; \mathrm{H}_{2}-1903 ; \mathrm{C}_{2} \mathrm{H}_{6}-14$; CO$180 ; \mathrm{CO}_{2}-635$.

Step 1. TDCG in $\mathrm{ppm}=2303$. TDCG is above normal (>720 ppm).

Step 2. The transformer is sampled again on $21 / 03 / 2009$ to determine rate of TDCG. Concentrations of dissolved gases in ppm are as follows: $\mathrm{C}_{2} \mathrm{H}_{2}-26 \mathrm{C}_{2} \mathrm{H}_{4}-23 ; \mathrm{CH}_{4}-2221 ; \mathrm{H}_{2}-2257$; $\mathrm{C}_{2} \mathrm{H}_{6}-22 ; \mathrm{CO}-220 ; \mathrm{CO}_{2}-821$.

TDCG in ppm = 2769; rate of TDCG $=3.10 \mathrm{lit} / \mathrm{day}$, which is greater than the normal level (2.8 lit/day).

Step 3. FIS is applied for fault diagnosis. The output of FIS is given by rule viewer which is shown in Figure 5. Rule viewer shows $R 1=1.13$ (Med), $R 2=0.0979$, which lies on the boundary of the fuzzy ratios Low and Med, and $R 3=1.05$ which lies on the boundary of the fuzzy ratios Low and Med. Dark dots in the fault column show that the rules 9, 10, 11, and 13 are satisfied which indicates possible faults PD and D1. This result matches with the actual fault of the transformer. Weighted average of both rules is given as 0.529 . Weight of both faults can be calculated as follows:

$$
\text { weight of } \mathrm{PD}=\frac{(0.6-0.529)}{(0.6-0.4)}=0.355 \text {. }
$$

$$
\text { weight of D1 }=1-0.355=0.645 \text {. }
$$

The weights point towards the strong possibility of fault D1 and the relatively less possibility of fault PD.

Step 4. Severity of faults is Medium. Maintenance actions suggested are as follows.

(1) Observe caution

(2) Retest oil monthly.

(3) Determine load dependence. 


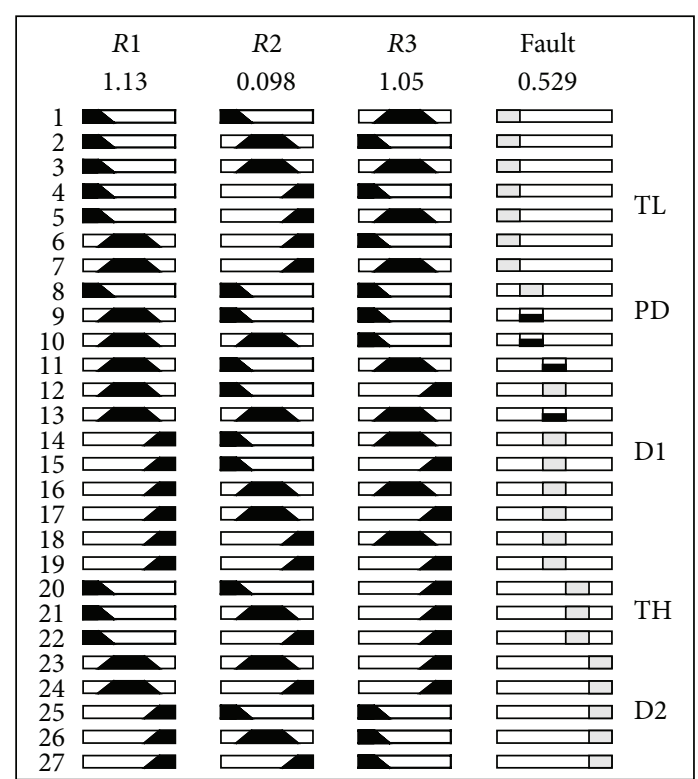

Figure 5: Rule viewer for case-III.

\section{Conclusion}

The proposed FIS is developed using "MATLAB". It can diagnose the incipient faults of the suspected transformers and suggest proper maintenance actions. The fuzzy threeratio method is proposed to diagnose multiple faults and faults that cannot be diagnosed by the conventional DGA methods. Proposed FIS provides fault diagnosis for all the cases. Accuracy of the proposed method is better than that of other diagnostic methods.

\section{References}

[1] I. Höhlein, "Unusual cases of gassing in transformers in service," IEEE Electrical Insulation Magazine, vol. 22, no. 1, pp. 24-27, 2006.

[2] C. Mayoux, "Degradation of insulating materials under electrical stress," IEEE Transactions on Dielectrics and Electrical Insulation, vol. 7, no. 5, pp. 590-601, 2000.

[3] M. K. Pradhan, "Assessment of the status of insulation during thermal stress accelerated experiments on transformer prototypes," IEEE Transactions on Dielectrics and Electrical Insulation, vol. 13, no. 1, pp. 227-237, 2006.

[4] I. Fofana, J. Sabau, D. Bussières, and E. B. Robertson, "The mechanism of gassing in power transformers," in Proceedings of the IEEE International Conference on Dielectric Liquids (ICDL '08), pp. 1-4, July 2008.

[5] V. G. Arakelian, "Effective diagnostics for oil-filled equipment," IEEE Electrical Insulation Magazine, vol. 18, no. 6, pp. 26-38, 2002.

[6] M. Agah, G. R. Lambertus, R. Sacks, and K. Wise, "High-speed MEMS-based gas chromatography," Journal of Microelectromechanical Systems, vol. 15, no. 5, pp. 1371-1378, 2006.

[7] M. Agah, G. Lambertus, R. Sacks, K. D. Wise, and J. A. Potkay, "High-performance temperature-programmed microfabricated gas chromatography columns," Journal of Microelectromechanical Systems, vol. 14, no. 5, pp. 1039-1050, 2005.
[8] M. Duval, "New techniques for dissolved gas-in-oil analysis," IEEE Electrical Insulation Magazine, vol. 19, no. 2, pp. 6-15, 2003.

[9] V. G. Arakelian, "The long way to the automatic chromatographic analysis of gases dissolved in insulating oil," IEEE Electrical Insulation Magazine, vol. 20, no. 6, pp. 8-25, 2004.

[10] N. Lelekakis, D. Martin, W. Guo, and J. Wijaya, "Comparison of dissolved gas-in-oil analysis methods using a dissolved gas-inoil standard," IEEE Electrical Insulation Magazine, vol. 27, no. 5, pp. 29-35, 2011.

[11] "Mineral oil-impregnated electrical equipment in service guide to the interpretation of dissolved and free gases analysis," IEC Publication 60599, 2007.

[12] X. Li, H. Wu, and D. Wu, "DGA interpretation scheme derived from case study," IEEE Transactions on Power Delivery, vol. 26, no. 2, pp. 1292-1293, 2011.

[13] "IEEE guide for interpretation of gases generated in oil immersed transformer," ANSI/IEEE Standard C57.104.2008, 2009.

[14] T. K. Saha, "Review of modern diagnostic techniques for assessing insulation condition in aged transformers," IEEE Transactions on Dielectrics and Electrical Insulation, vol. 10, no. 5, pp. 903-917, 2003.

[15] N. A. Muhamad, B. T. Phung, T. R. Blackburn, and K. X. Lai, "Comparative study and analysis of DGA methods for transformer mineral oil," in Proceedings of the IEEE Lausanne Power Tech, pp. 45-50, July 2007.

[16] M. Duval and A. dePablo, "Interpretation of gas-in-oil analysis using new IEC publication 60599 and IEC TC 10 databases," IEEE Electrical Insulation Magazine, vol. 17, no. 2, pp. 31-41, 2001.

[17] M. Duval and J. J. Dukarm, "Improving the reliability of transformer gas-in-oil diagnosis," IEEE Electrical Insulation Magazine, vol. 21, no. 4, pp. 21-27, 2005.

[18] M. Duval, "A review of faults detectable by gas-in-oil analysis in transformers," IEEE Electrical Insulation Magazine, vol. 18, no. 3, pp. 8-17, 2002.

[19] M. Duval, "The duval triangle for load tap changers, nonmineral oils and low temperature faults in transformers," IEEE Electrical Insulation Magazine, vol. 24, no. 6, pp. 22-29, 2008.

[20] C. E. Lin, J. M. Ling, and C. L. Huang, "Expert system for transformer fault diagnosis using dissolved gas analysis," IEEE Transactions on Power Delivery, vol. 8, no. 1, pp. 231-238, 1993.

[21] Y.-C. Huang, H.-T. Yang, and C.-L. Huang, "Developing a new transformer fault diagnosis system through evolutionary fuzzy logic," IEEE Transactions on Power Delivery, vol. 12, no. 2, pp. 761-767, 1997.

[22] H.-T. Yang and C.-C. Liao, "Adaptive fuzzy diagnosis system for dissolved gas analysis of power transformers," IEEE Transactions on Power Delivery, vol. 14, no. 4, pp. 1342-1350, 1999.

[23] Y. Zhang, X. Ding, Y. Liu, and P. J. Griffin, "An artificial neural network approach to transformer fault diagnosis," IEEE Transactions on Power Delivery, vol. 11, no. 4, pp. 1836-1841, 1996.

[24] J. L. Guardado, J. L. Naredo, P. Moreno, and C. R. Fuerte, "A comparative study of neural network efficiency in power transformers diagnosis using dissolved gas analysis," IEEE Transactions on Power Delivery, vol. 16, no. 4, pp. 643-647, 2001.

[25] D. V. S. S. Siva Sarma and G. N. S. Kalyani, "ANN approach for condition monitoring of power transformers using DGA," in Proceedings of the IEEE Region 10 Conference (TENCON '04), vol. 3, pp. C444-C447, November 2004. 
[26] W. M. Lin, C. H. Lin, and M. X. Tasy, “Transformer-fault diagnosis by integrating field data and standard codes with training enhancible adaptive probabilistic network," IEE Generation Transmission Distribution, vol. 152, no. 3, pp. 335-341, 2005.

[27] Y.-C. Huang, "Evolving neural nets for fault diagnosis of power transformers," IEEE Transactions on Power Delivery, vol. 18, no. 3, pp. 843-848, 2003.

[28] R. Naresh, V. Sharma, and M. Vashisth, "An integrated neural fuzzy approach for fault diagnosis of transformers," IEEE Transactions on Power Delivery, vol. 23, no. 4, pp. 2017-2024, 2008.

[29] D. Bhalla, R. K. Bansal, and H. O. Gupta, "Application of artificial intelligence techniques for Dissolved Gas Analysis of transformers-a review," World Academy of Science, Engineering and Technology, vol. 62, pp. 221-229, 2010.

[30] D. R. Morais and J. G. Rolim, "A hybrid tool for detection of incipient faults in transformers based on the dissolved gas analysis of insulating oil," IEEE Transactions on Power Delivery, vol. 21, no. 2, pp. 673-680, 2006.

[31] R. A. Hooshmand, M. Parastegari, and Z. Forghani, "Adaptive neuro-fuzzy inference system approach for simaltaneous diagnosis of the type and location of faults in power transformers," IEEE Electrical Insulation Magzine, vol. 28, no. 5, pp. 32-42, 2012.

[32] W. Chen, C. Pan, Y. Yun, and Y. Liu, "Wavelet networks in power transformers diagnosis using dissolved gas analysis," IEEE Transactions on Power Delivery, vol. 24, no. 1, pp. 187-194, 2009.

[33] Z. Wang, Y. Liu, and P. J. Griffin, "A combined ANN and expert system tool for transformer fault diagnosis," IEEE Transactions on Power Delivery, vol. 13, no. 4, pp. 1224-1229, 1998.

[34] Mathworks, Fuzzy Logic Toolbox, User's Guide.

[35] D. Driankov, H. Hellendoorn, and M. Reinfrank, An Introduction to Fuzzy Control, Narosa Publishing House, New Delhi, India.

[36] G. J. Klir and T. A. Folger, Fuzzy Sets, Uncertainity and Information, PHI, New Delhi, India.

[37] M. McNeill and E. Thro, Fuzzy Logic, A Practical Approach.

[38] "IEEE guide for dissolved gas analysis in transformer load tap changers," IEEE Standard C57.139-2010. 

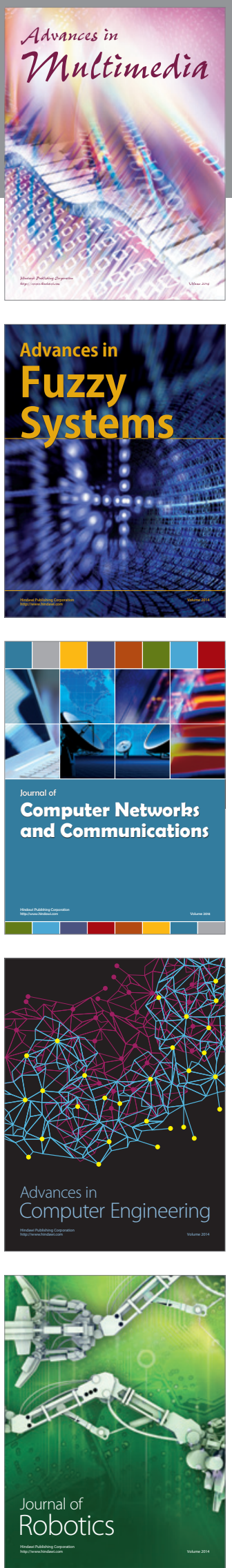

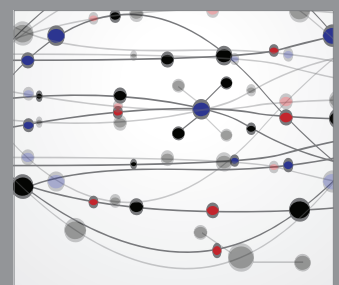

The Scientific World Journal
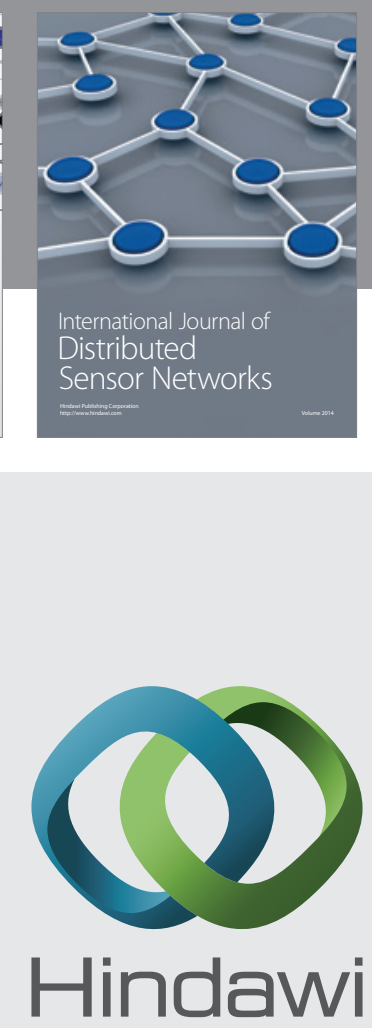

Submit your manuscripts at

http://www.hindawi.com
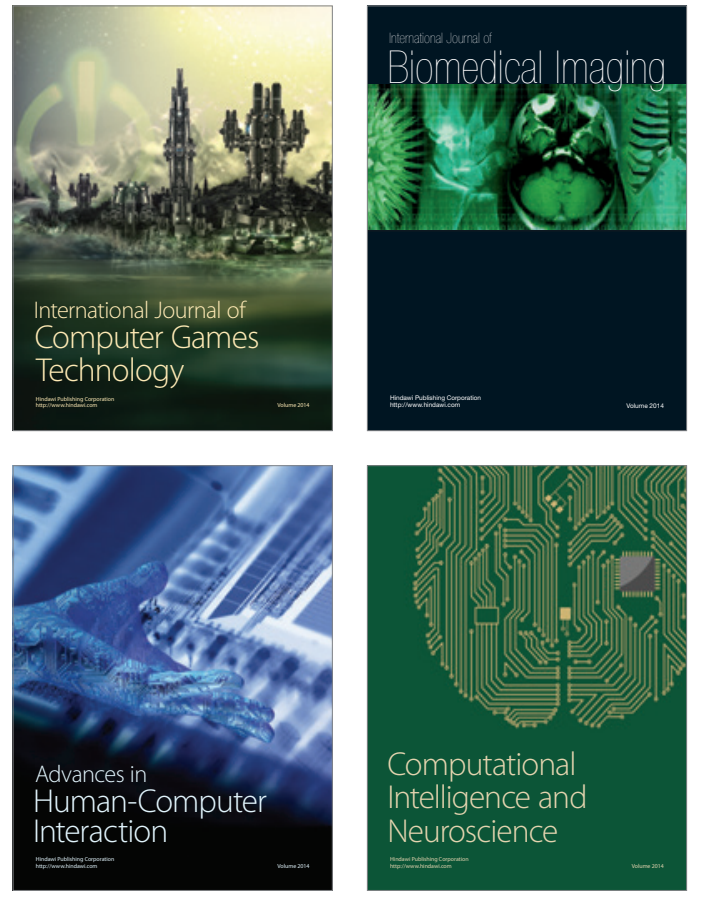
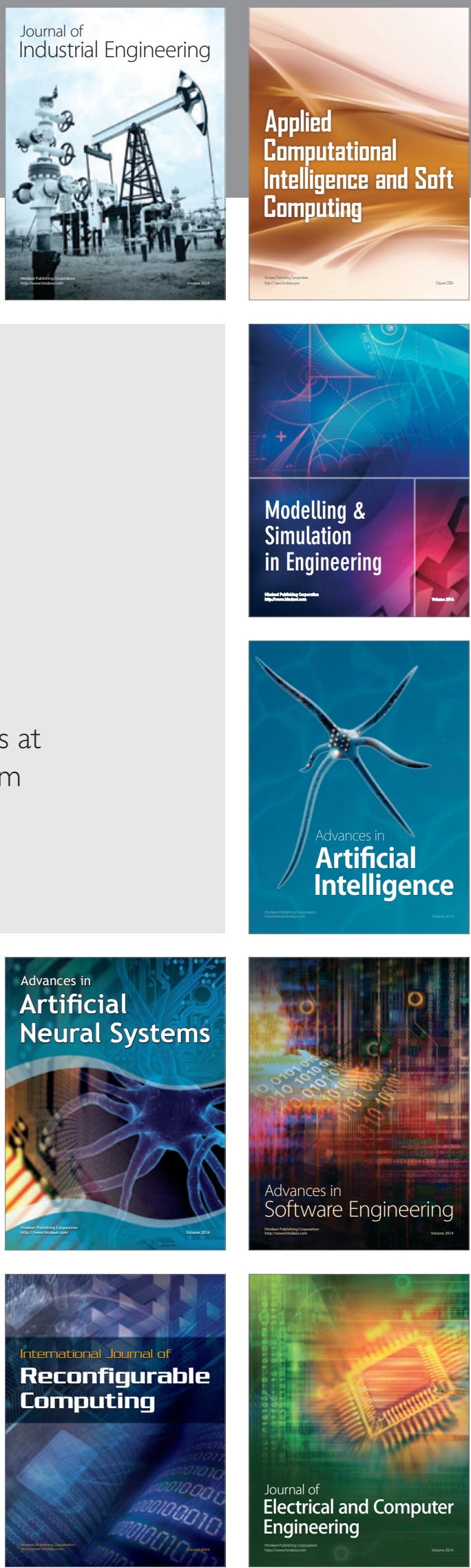